\title{
Withdrawal Symptoms after Selective Serotonin Reuptake Inhibitor Discontinuation: A Systematic Review
}

\author{
Giovanni A. Fava a, b Alessia Gatti ${ }^{a}$ Carlotta Belaise $^{a}$ Jenny Guidi ${ }^{a}$ \\ Emanuela Offidani ${ }^{c}$ \\ ${ }^{a}$ Affective Disorders Program, Department of Psychology, University of Bologna, Bologna, Italy; ${ }^{b}$ Department of \\ Psychiatry, State University of New York at Buffalo, Buffalo, N.Y., and ${ }^{\mathrm{C} C e n t e r}$ for Complementary and Integrative \\ Medicine, Weill Cornell Medical College, New York, N.Y., USA
}

\section{Key Words \\ Withdrawal symptoms · Selective serotonin reuptake inhibitors · Adverse events · Side effects · Discontinuation syndrome $\cdot$ Tolerance $\cdot$ Antidepressive agents $\cdot$ Depression . Anxiety}

\begin{abstract}
Background: Selective serotonin reuptake inhibitors (SSRI) are widely used in medical practice. They have been associated with a broad range of symptoms, whose clinical meaning has not been fully appreciated. Methods: The PRISMA guidelines were followed to conduct a systematic review of the literature. Titles, abstracts, and topics were searched using the following terms: 'withdrawal symptoms' OR 'withdrawal syndrome' OR 'discontinuation syndrome' OR 'discontinuation symptoms', AND 'SSRI' OR 'serotonin' OR 'antidepressant' OR 'paroxetine' OR 'fluoxetine' OR 'sertraline' OR 'fluvoxamine' OR 'citalopram' OR 'escitalopram'. The electronic research literature databases included CINAHL, the Cochrane Library, PubMed and Web-of-Science from inception of each database to July 2014. Results: There were 15 randomized controlled studies, 4 open trials, 4 retrospective investigations, and 38 case reports. The prevalence of the
\end{abstract}

syndrome was variable, and its estimation was hindered by a lack of case identification in many studies. Symptoms typically occur within a few days from drug discontinuation and last a few weeks, also with gradual tapering. However, many variations are possible, including late onset and/or longer persistence of disturbances. Symptoms may be easily misidentified as signs of impending relapse. Conclusions: Clinicians need to add SSRI to the list of drugs potentially inducing withdrawal symptoms upon discontinuation, together with benzodiazepines, barbiturates, and other psychotropic drugs. The term 'discontinuation syndrome' that is currently used minimizes the potential vulnerabilities induced by SSRI and should be replaced by 'withdrawal syndrome'.

(c) 2015 S. Karger AG, Basel

\section{Introduction}

In the 1990s, case reports [1-16] started documenting withdrawal reactions following the discontinuation of selective serotonin reuptake inhibitors (SSRI). As the volume of the literature grew, one of the earliest reviews [17] suggested that a discontinuation syndrome consisting of a cluster of somatic and psychological symptoms could

\section{KARGER 125}

(c) 2015 S. Karger AG, Base

0033-3190/15/0842-0072\$39.50/0

E-Mail karger@karger.com

www.karger.com/pps
Giovanni A. Fava, MD

Affective Disorders Program, Department of Psychology

University of Bologna, Viale Berti Pichat 5

IT-40127 Bologna (Italy)

E-Mail giovanniandrea.fava@unibo.it 
indeed occur. The clinical data suggested that the syndrome could last up to 3 weeks and could be improved by restarting the same or a similar antidepressant drug (AD). Even though discontinuation symptoms were mostly reported after abrupt suspension, they were found to occur also after gradual tapering [17-19] and to differ in prevalence according to the pharmacological profile of SSRI $[20,21]$.

In 1998, a specific index for the withdrawal symptomatology, the Discontinuation Emergent Signs and Symptoms (DESS) checklist [22], was developed. Diagnostic criteria, such as those developed by Black et al. [23], were proposed. Such criteria encompassed both somatic (dizziness, light-headedness, vertigo, shock-like sensations, paresthesias, fatigue, headache, nausea, tremor, diarrhea, and visual disturbances) and psychological (anxiety, insomnia, and irritability) symptoms and required significant distress to be associated with them [23].

The term 'discontinuation syndrome' has progressively replaced 'withdrawal syndrome' or 'reactions' [24]. In the past decade, few studies assessed the presence of discontinuation symptoms, and the topic has attracted limited attention also as to literature reviews [25-28]. To the best of our knowledge, in the English language there has been no systematic review on the clinical aspects of SSRI discontinuation according to established criteria [29]. Yet, such knowledge is important because of the widespread use of SSRI in medical practice.

\section{Methods}

\section{Data Sources}

The PRISMA guidelines were followed to conduct a systematic review of the literature, examining the presence of SSRI withdrawal/discontinuation symptoms [29]. Titles, abstracts, and topics were searched using the following terms: 'withdrawal symptoms' OR 'withdrawal syndrome' OR 'discontinuation syndrome' OR 'discontinuation symptoms', AND 'SSRI' OR 'serotonin' OR 'antidepressant' OR 'paroxetine' OR 'fluoxetine' OR 'sertraline' OR 'fluvoxamine' OR 'citalopram' OR 'escitalopram'. The electronic research literature databases included CINAHL, the Cochrane Library, PubMed, and Web-of-Science from inception of each database to July 2014. Limits were set to adult population, humans, and English language. In addition, reference lists and citations of initially identified reports were examined. Additional clinical trials were searched manually.

\section{Study Selection}

Search was carried out independently by two investigators (A.G. and C.B.); disagreements were resolved by consensus among these primary raters and the other investigators (G.A.F., J.G., and E.O.). Articles were considered to be eligible if they reported at least one case of withdrawal syndrome after the discontinuation of
SSRI. We restricted our search to SSRI drugs. Due to the importance of serendipitous observations, we included studies of various designs: randomized controlled trials (RCT), open investigations, retrospective studies, and single case reports. We excluded studies if they were conducted in nonclinical samples and settings and/or were based on the exploration of patients' websites [30].

\section{Data Extraction}

Data were independently extracted by both reviewers with the use of a precoded form. The following data were extracted from studies meeting the criteria for inclusion in the systematic review: age, gender distribution, study design, type of SSRI, number of patients, group comparisons, treatment duration and assessment times, and methods used to assess withdrawal symptoms. The terms 'relapse/recurrence', 'rebound', and 'withdrawal' were used according to Chouinard's definitions [31]: recurrent symptoms are a gradual return of the patient's original symptoms with the same rate of intensity as before treatment; rebound symptoms are a rapid return of the original symptoms but worse than before treatment, and withdrawal symptoms are new symptoms that were not part of the patient's illness before treatment and are associated with specific classes of psychotropic drugs. The terms 'withdrawal syndromes' and 'discontinuation syndromes' were considered to be equivalent. Particularly in case reports, adherence to the prototype description of SSRI discontinuation syndromes [23] was checked but was not considered to be a requisite.

\section{Data Synthesis}

In view of the highly heterogeneous patient populations and differences in the design of RCT, a meta-analysis was not deemed to be appropriate.

\section{Results}

\section{Characteristics of Included Studies}

The literature search identified 437 potentially relevant papers about SSRI withdrawal/discontinuation syndrome; 275 were left after the removal of duplicates. From the titles and abstracts, 31 studies were excluded since they involved psychotropic drugs other than SSRI. Further exclusion involved 128 publications due to their lack of pertinence to withdrawal reactions and 55 others for being reviews or commentaries. A total of 61 investigations met the criteria for inclusion in the study (online suppl. fig. 1, for all online suppl. material, see www.karger.com/doi/10.1159/000370338). There were 15 RCT comparing fluoxetine, sertraline, paroxetine, citalopram, escitalopram, and placebo or other $\mathrm{AD}$ (online suppl. table 1). Other studies included 4 open trials and 4 retrospective investigations (online suppl. table 2). A total of 38 clinical reports were also examined (online suppl. table 3 ). These studies were summarized and critically reviewed according to the characteristics of the research design. 
Discontinuation Symptoms in RCT

Single Drug and Placebo Comparisons

The first RCT assessing the presence of discontinuation symptoms after SSRI suspension was conducted in 120 patients suffering from panic disorder and who were treated with cognitive therapy and paroxetine $(20,40,60 \mathrm{mg}$ /day) for 12 weeks [32]. After abrupt discontinuation, withdrawal symptoms (mostly dizziness) occurred in a significantly greater proportion of individuals taking paroxetine (34.5\%) than in subjects receiving placebo (13.5\%) [32]. In 1998, Zajecka et al. [17] treated 395 patients meeting the DSMIII-R criteria for major depression with 12 weeks of fluoxetine. Patients who responded were randomly assigned to placebo (abrupt discontinuation) or continuation for 6 weeks. Discontinuation symptoms were assessed by spontaneous reports and open-ended questions. After 2 weeks, somnolence was found to be significantly more frequently present in patients who abruptly discontinued the medication $(4 \%)$ than in those who continued to receive fluoxetine $(0 \%, \mathrm{p}<0.01)$. At weeks 4 and 6 , subjects in the placebo group reported dizziness more frequently than patients in the continuation group ( 7 vs. $4 \%, \mathrm{p}<0.05$ and 5 vs. $1 \%, \mathrm{p}<0.05$, respectively).

In 2000, Markowitz et al. [33] reexamined data from a previously conducted placebo-controlled trial [34] which assessed the efficacy of citalopram in patients with depression. After 8 weeks of treatment, patients were randomized to receive placebo (discontinuation condition) or to continue with the active drug for another 24 weeks. Discontinuation symptoms were assessed after 2 weeks by using the unwanted side effect scale (UKU) [35]. Results, obtained according to the patients' relapse status, showed no differences in neurological and psychiatric symptoms between individuals switched to placebo and those who continued with the active treatment. However, the duration of treatment was brief, and the assessment methods were not geared to detecting withdrawal symptoms.

Montgomery et al. [36], after a 12-week open-label treatment with escitalopram, randomized 371 patients suffering from social anxiety disorder $(\mathrm{SAD})$ to a double-blind controlled study comparing escitalopram (from 10 to 20 $\mathrm{mg} /$ day; $\mathrm{n}=190)$ and placebo $(\mathrm{n}=181)$. Results showed that, 1 and 2 weeks after randomization, the total DESS score was significantly higher in those who discontinued escitalopram than in the continuation group $(\mathrm{p}<0.01)$.

\section{Multiple Drug Comparisons}

Several studies comparing different SSRI investigated the onset of discontinuation symptoms. In 1998, Rosenbaum et al. [22] conducted a 1-week randomized double- blind placebo substitution trial to assess the emergence of discontinuation symptoms in 231 patients with major depressive disorder (MDD) treated with paroxetine, sertraline, or fluoxetine. After treatment interruption, discontinuation symptoms, as measured through the DESS, were more frequently reported by patients treated with paroxetine $(\mathrm{p}<0.001)$ and sertraline $(\mathrm{p}<0.001)$ than by those receiving fluoxetine. When treatments were compared, individuals treated with paroxetine reported the greatest number of DESS symptoms compared to those treated with sertraline $(\mathrm{p}<0.05)$ and fluoxetine $(\mathrm{p}<$ $0.001)$. Further, during discontinuation, anxiety, depression, somatic symptoms, and hostility were significantly greater in the paroxetine and sertraline groups $(\mathrm{p}<0.001$ in all conditions) than in the fluoxetine arm.

In a subsequent study, Michelson et al. [37] recruited patients with a history of depression, successfully treated with fluoxetine, sertraline, or paroxetine. At entry, patients had been taking medication continuously for at least 4 months but not more than 3 years and had had no dose changes for the 2 months prior to study entry. Under double-blind order-randomized conditions, all subjects underwent placebo substitution during a 5-day period and continued treatment with their usual SSRI during the next 5-day period. Subjects continued treatment with the SSRI at all other times. The study showed that placebo substitution, but not continued active medication, was associated with statistically significant increases in total numbers of solicited adverse events for patients treated with paroxetine by the end of the fourth day $(p<0.001)$. Increases in symptoms for patients treated with paroxetine became statistically significant as early as at the time of the second dose of placebo [37].

Judge et al. [38] reached similar results comparing paroxetine and fluoxetine during a 5- to 8-day randomized placebo substitution. About $16 \%$ of patients taking paroxetine versus $6 \%$ of those receiving fluoxetine reported discontinuation symptoms $(\mathrm{p}<0.05)$. During discontinuation, the paroxetine treatment group also reported significant increases in depressive symptoms and clinical global severity scores as well as difficulty in social functioning. Paroxetine resulted also in significantly higher scores on the DESS when compared to agomelatine in 192 patients with MDD [39]. Rates of withdrawal symptoms were significantly greater in patients who discontinued paroxetine than in those continuing on medication $(\mathrm{p}<$ 0.001 ), while no significant differences were found in the agomelatine patient group $(3.0 \pm 4.2$ and $4.4 \pm 5.7)$.

Similar results emerged also when paroxetine was directly compared to other SSRI. Specifically, Hindmarch
74

Psychother Psychosom 2015;84:72-81 DOI: $10.1159 / 000370338$
Fava/Gatti/Belaise/Guidi/Offidani 
et al. [40], in 87 patients suffering from depression who had their maintenance therapy with paroxetine, fluoxetine, sertraline, and citalopram interrupted for 4-7 days, reported that, although DESS scores increased in all treatment groups, paroxetine caused significantly more discontinuation symptoms than other SSRI did $(\mathrm{p}<0.05)$.

Two RCT examined the onset of withdrawal symptoms following treatment with escitalopram [41] or sertraline [42]. When escitalopram was compared to venlafaxine XR in MDD patients, significantly more venlafaxine-treated patients than escitalopram-treated ones reported a DESS score $>4$ in a 1-week runout period $(\mathrm{p}<$ 0.01 ) [41]. Increased dreaming, trouble sleeping, nervousness or anxiety, irritability, and sudden worsening of mood occurred with an incidence $>10 \%$ in the escitalopram group. Sir et al. [42] systematically evaluated the presence of withdrawal reactions by using the Antidepressant Discontinuation Scale (ADDS) [42] in an 8-week multicenter randomized trial comparing sertraline and venlafaxine XR in 163 patients with MDD. After a tapered discontinuation, $34.3 \%$ of patients treated with sertraline experienced a discontinuation syndrome from moderate to severe; $23.9 \%$ of them experienced a mild discontinuation reaction, while $23.9 \%$ reported a minimal one. Subjects taking venlafaxine $\mathrm{XR}$ also reported discontinuation symptoms from minimal to very severe ( $\mathrm{minimal}=17.7 \%$; mild $=27.4 \%$; moderate $=38.7 \%$; severe $=3.2 \%$; very severe $=1.6 \%$ ). The authors reported only ADDS symptoms that occurred in more than $10 \%$ of patients, showing no differences between those who received sertraline compared to venlafaxine XR. Dizziness (33.3\%), vivid dreams $(26.4 \%)$, fatigue (22.2\%), vertigo (5.6\%), rapid changing mood $(6.9 \%)$, and tachycardia $(4.2 \%)$ were the most frequently reported symptoms [42].

Paroxetine was observed to cause discontinuation symptoms also in patients with anxiety disorders. In SAD patients, $28.4 \%$ of those who were treated with paroxetine had a total DESS score $\geq 4$, compared to 15.1, 17.1, and $21.7 \%$ of patients treated with escitalopram $(5,10$, and 15 $\mathrm{mg}$, respectively) and $1.9 \%$ of patients assigned to placebo ( $p<0.001$ vs. placebo) [43]. Similar results emerged in patients suffering from generalized anxiety disorder. After a 12-week randomized placebo-controlled trial of escitalopram $(5,10$, or $20 \mathrm{mg}$, respectively) and paroxetine, generalized anxiety disorder patients underwent a washout period of 2 weeks [44]. At discontinuation, while patients treated with escitalopram did not differ in terms of adverse events from placebo controls, those who discontinued paroxetine showed a significantly higher mean DESS score compared to controls $(\mathrm{p}<0.001)$. As to active drugs, paroxetine produced significantly more discontinuation symptoms than escitalopram did ( 5 or $10 \mathrm{mg}$, p < 0.001) [44].

Baldwin et al. [45] evaluated the difference between abrupt and tapered discontinuation when using paroxetine or escitalopram in patients with MDD. Discontinuation-emergent effects were assessed in two separate double-blind periods. First, patients were randomized to one of two treatment interruption periods (placebo substitution for 3-5 days), and then they were randomized to a 1- to 2-week tapered withdrawal period. During the short placebo substitution, there were no significant differences. In the tapered condition, results showed that the changes in the total DESS score were significantly greater for paroxetine than escitalopram after 1 week of alternate-day dosing $(\mathrm{p}<0.01)$ and a subsequent (posttreatment) week with placebo $(\mathrm{p}<0.01)$. In order to examine two different tapering strategies, Tint et al. [46] randomized 28 patients treated with different SSRI or venlafaxine to a short (3 days) or long (14 days) taper. Results showed that $46 \%$ of subjects reported discontinuation symptoms and that the incidence was similar in both conditions ( 47 vs. $46 \%$ in the short vs. long taper groups).

In conclusion, among patients with MDD, the average mean DESS score was 5.7 ( $\mathrm{SD}=7.36)$ for those who discontinued treatment with paroxetine [22, 38, 39, 45], whereas for escitalopram $[41,45]$ it ranged from 2.4 to 3.2 , and in both studies investigating fluoxetine discontinuation $[22,38]$, it was $0.2(\mathrm{SD}=3.86)$. In patients with $\mathrm{SAD}$, rates of discontinuation symptoms after treatment with escitalopram (10-20 mg/day) ranged from 17 to $27 \%$ $[36,43]$. However, only a few studies used either a definition of withdrawal reaction based on specific criteria $[23$, $47]$ or a cutoff score of the DESS [36, 40, 43, 46] to determine the occurrence of withdrawal symptoms.

\section{Withdrawal Symptoms in Open Trials}

Two open trials of fluvoxamine involving respectively 17 obsessive-compulsive disorder [48] and 14 panic disorder [3] patients systematically reported the emergence of withdrawal syndromes. In both reports, patients experienced withdrawal reactions as assessed by using the 90 item Hopkins Symptom Checklist (HSCI-90) [48] or relying on spontaneous reports [3]. Twenty-three percent of obsessive-compulsive disorder patients [48] and $85.7 \%$ of PD patients [3] experienced dizziness, nausea, headache, confusion, low energy, and weakness that abated in about 2-3 weeks.

Bogetto et al. [49] examined the discontinuation syndrome in 97 patients with dysthymic disorder treated 
with either paroxetine $(n=52)$ or fluoxetine $(n=45)$. About $27 \%$ of the total sample reported withdrawal symptoms after discontinuing their medication according to their psychiatrist's instructions. Of these, $84.6 \%$ were treated with paroxetine. Further, patients with an earlier onset of dysthymic disorder were more likely to report withdrawal symptoms.

An investigation by Fava et al. [50] explored the prevalence and features of discontinuation syndromes ensuing with gradual tapering of SSRI in patients with panic disorder and agoraphobia who had remitted upon behavioral treatment. Nine of 20 patients (45\%) experienced a discontinuation syndrome according to specific criteria based on the DESS. Discontinuation syndromes subsided within a month in all but 3 patients (27\%). These 3 patients all received paroxetine and displayed alternation of worsened mood, fatigue, and emotional instability with trouble sleeping, irritability, and hyperactivity, meeting the DSM-IV criteria [51] for cyclothymic disorder except for duration.

\section{Withdrawal Symptoms in Retrospective Studies}

Coupland et al. [52] used retrospective charts to report the incidence of withdrawal symptoms in 171 patients who discontinued the treatment with clomipramine and different SSRI (fluoxetine, fluvoxamine, paroxetine, and sertraline). Symptoms occurred significantly more frequently in patients who were treated either with one of the shorter half-life SSRI, fluvoxamine or paroxetine (17.2\%), or with clomipramine $(30.8 \%)$ than in patients taking SSRI with longer half-life metabolites such as sertraline or fluoxetine (1.5\%). In a similar study [53], the UK database for spontaneous reports of suspected adverse drug reactions has been used to describe the reactions associated with the discontinuation of different SSRI. Authors pointed out that withdrawal reactions with paroxetine constituted a greater proportion of the reports than with the other SSRI. Discontinuation syndrome was also more common in young patients than in the elderly.

Himei and Okamura [54] investigated the occurrence of discontinuation symptoms by using clinical records of 385 outpatients who were diagnosed with a single episode of MDD and had undergone monotherapy with paroxetine in the previous 5 years. Findings showed that $10 \%$ of patients experienced discontinuation symptoms. Furthermore, withdrawal symptoms were more likely to occur in patients who discontinued paroxetine abruptly than in those who tapered the drug. Interestingly, an association between the occurrence of discontinuation syndrome and age emerged, although such an associa- tion seemed to be moderated by the fact that younger patients were more inclined to abruptly stop the medication.

Van Geffen et al. [55] investigated different tapering strategies by evaluating retrospectively features of discontinuation in patients who had previously interrupted their SSRI. A significant difference emerged favoring a tapered discontinuation. Specifically, patients who suspended their medication abruptly reported a significantly greater number of adverse events than those who tapered the drug $(\mathrm{p}<0.01)$.

\section{Withdrawal Symptoms in Case Reports}

Fluoxetine

Six case reports of fluoxetine withdrawal reactions were retrieved [1, 5, 10, 56-58]. In all cases, subjects abruptly discontinued their medications, and withdrawal symptoms mostly appeared within 2 days, except for 1 patient who reported symptoms 2 weeks after discontinuation. Common symptoms were dizziness, light-headedness, and sleep disturbances. Two subjects also experienced delirium $[10,58]$. One patient complained about dystonic reactions [1], and 1 experienced prolonged rebound cataplexy [57]. The authors did not report information on the duration of the withdrawal syndromes, probably because symptoms were treated reintroducing fluoxetine $[10,57,58]$ or administering other drugs $[1,5]$.

\section{Paroxetine}

We identified 18 case reports of withdrawal symptoms in adult patients (aged 22-73 years) taking paroxetine. Discontinuation symptoms were reported independently of treatment duration (from few weeks to years) and type of discontinuation (abrupt vs. tapered). Symptoms usually appeared 1 week after discontinuation, except for 1 patient who experienced symptoms after 1 month [59]. The most commonly reported symptoms were dizziness, light-headedness, fatigue, sleep disturbance, and gastrointestinal disturbance $[7,11,60]$. Less common reactions were 'electric-shock' sensation $[9,59,61,62]$, visual and auditory hallucination [63], nocturnal enuresis [64], pruritus [65], and emesis [6]. In most reports, symptoms lasted until paroxetine was reintroduced or switched to other medications and/or cognitive behavioral therapy [12, $13,15,59,62,66]$. Only in a few cases did symptoms spontaneously remit in about 2 weeks $[4,8,63,67]$.

\section{Sertraline}

Eight case reports highlighted the presence of withdrawal reactions in subjects discontinuing sertraline. In 
Table 1. Signs and symptoms of withdrawal from SSRI

\begin{tabular}{ll}
\hline System involved & Symptoms \\
\hline General & Flu-like symptoms, fatigue, weakness, tiredness, headache, tachycardia, dyspnea \\
Balance & Gait instability, ataxia, dizziness, light-headedness, vertigo \\
Sensory & Paresthesias, electric-shock sensations, myalgias, neuralgias, tinnitus, altered taste, pruritus \\
Visual & Visual changes, blurred vision \\
Neuromotor & Tremor, myoclonus, ataxia, muscle rigidity, jerkiness, muscle aches, facial numbness \\
Vasomotor & Sweating, flushing, chills \\
Sleep & Insomnia, vivid dreams, nightmares, hypersomnia, lethargy \\
Gastrointestinal & Nausea, vomiting, diarrhea, anorexia, abdominal pain \\
Affective & Anxiety, agitation, tension, panic, depression, intensification of suicidal ideation, \\
& irritability, impulsiveness, aggression, anger, bouts of crying, mood swings, derealization \\
Psychotic & and depersonalization \\
Cognitive & Visual and auditory hallucinations \\
Sexual & Confusion, decreased concentration, amnesia \\
\hline
\end{tabular}

all reports, withdrawal reactions appeared within 1-5 days following either abrupt $[68,69]$ or tapered $[9,14,13$, $16,70,71]$ discontinuation and lasted from 1-2 weeks $[13,14,69,70]$ to 6 weeks [16] or even longer (13-14 weeks) $[9,68]$. In most cases, symptoms disappeared spontaneously, except for 2 reports in which sertraline $25-50 \mathrm{mg} /$ day was reintroduced $[68,71]$ and another 2 in which a combination of psychotropic drugs was prescribed $[69,70]$. Fatigue, instability, vertigo, and flu-like symptoms were the more frequently reported adverse reactions. Extrapyramidal symptoms and electric-shock sensations were also reported $[9,70]$. Furthermore, 1 patient experienced orthostatic hypotension [71].

Fluvoxamine

Three case reports of fluvoxamine discontinuation syndrome were also published $[2,72,73]$. Even though one patient gradually discontinued fluvoxamine [72] and another missed only few doses [73], they both experienced withdrawal symptoms such as dizziness, nausea, paresthesia, loss of concentration, restless feelings, and agitation. Symptoms were managed with fluvoxamine resumption $[2,73]$ or treated with clonazepam [72].

\section{Citalopram}

Three clinical cases reporting withdrawal reactions in patients receiving citalopram were also found [74-76]. All subjects showing withdrawal syndromes gradually discontinued citalopram. Symptoms appeared within 1-5 days after the last dose and lasted from 1 week [74] to 2 months [76]. Common symptoms were dizziness, anxiety, and irritability. One subject also experienced hypersensitivity of the genitals and premature ejaculation [76].

\section{Escitalopram}

Case reports of escitalopram discontinuation syndrome involved 2 patients $[77,78]$. One subject gradually reduced escitalopram (over 3 weeks) [77], while the other first missed a few doses and then reduced the drug very gradually (over 9 months) [78]. Both experienced electric-shock sensations a few days after drug stoppage, which lasted from 5 days [77] to 4 weeks [78].

\section{Discussion}

Despite the limited literature available, the results of this systematic review indicate that withdrawal symptoms may occur with any type of SSRI (citalopram, escitalopram, fluoxetine, fluvoxamine, paroxetine, and sertraline), even though they are exceedingly more frequent with paroxetine. Symptoms when paroxetine was discontinued were significantly superior to placebo in most of the RCT that were performed [22, 32, 37-40, 43-45]. The duration of treatment might be as short as 2 months [33, $39,42]$. The prevalence of the syndrome was variable, and its estimation was hindered by a lack of case identification in many studies. Gradual tapering does not eliminate the risk of withdrawal reactions. Indeed, a significant advantage of gradual tapering compared to abrupt discontinuation did not emerge $[45,46]$. A wide range of clinical 
manifestations encompassing both physical and psychological symptoms (summarized in table 1) may ensue. Such symptoms require adequate methods of detection (such as DESS) and specific questioning. There are many similarities with withdrawal phenomena that characterize second-generation antidepressants such as venlafaxine and duloxetine $[79,80]$ and benzodiazepines [27] but more limited overlap with those occurring with tricyclic antidepressants [81].

The withdrawal syndrome typically occurs within a few days from drug discontinuation and lasts a few weeks. However, many variations are possible, including late onset and/or longer persistence of disturbances $[9,16,68$, 76]. Bhanji et al. [59] and Fava et al. [50] documented the persistence of symptoms up to 1 year following paroxetine discontinuation. Belaise et al. [62] described 3 cases of what they defined as 'persistent post-withdrawal disorders induced by paroxetine'. Such disturbances appear to be quite common on patients' websites [30] but await adequate exploration in clinical studies.

The pathophysiological mechanisms of withdrawal phenomena are still hypothetical and in need of proper neurobiological investigations. A number of mechanisms have been suggested: a decrease in serotonin availability when treatment ends [82], a behavioral stress response that is associated with increased hippocampal N-methyl-D-aspartate receptor density [83], and genetic vulnerabilities [84]. Animal studies concerned with the longterm use of $\mathrm{AD}$ have limited implications, since clear discontinuation-like behaviors in animal models are not available [28]. Fava and Offidani [85] have interpreted the withdrawal phenomena according to the oppositional model of tolerance. Continued drug treatment may recruit processes that oppose the initial acute effects of a drug and may lead to a loss of efficacy and/or to a treatment-unresponsive course. When drug treatment ends, oppositional processes may operate for some time, resulting in the appearance of withdrawal symptoms and/or an increased vulnerability to relapse and/or resistance to treatment (if it is reinstituted) and/or switching to a bipolar course [85]. The appearance of withdrawal symptoms after SSRI discontinuation may thus be potentially related to a number of important clinical phenomena [86].

An editorial published in the late 1990s claimed that AD discontinuation reactions were preventable and simple to treat [47]. The evidence that we have just collected indicates the contrary.

First, gradual tapering of SSRI appears to be a reasonable clinical strategy but does not prevent the onset of withdrawal phenomena. We do not have a clear and spe- cific set of sociodemographic and clinical characteristics that may be associated with increased vulnerability.

Second, recognition of withdrawal symptoms requires careful exploration of the potential symptomatology (table 1) through specific questioning and/or instruments and knowledge of the pretreatment status. Symptoms may be easily misidentified as signs of impending relapse. Withdrawal symptoms are likely to have an early onset, while recurrent symptoms generally present with a gradual return. However, this is only a general tendency with mood disorders and not a rule, which in any event does not apply to the widespread use of SSRI in anxiety disorders [87]. The clinical picture may be complicated by the fact that rebound phenomena (a rapid return and worsening of the patient's original symptoms) may also take place with SSRI [86].

Third, discontinuation of SSRI may trigger complex phenomena, which may or may not be related to the onset of withdrawal symptomatology, such as hypomania or mania $[85,88]$ and persistent postwithdrawal disorders [62]. The term iatrogenic comorbidity refers to the lasting effects that previous treatments may entail (e.g., mood instability and high reactivity to environmental stimuli in persistent postwithdrawal disorders), well beyond their time of administration $[89,90]$.

Fourth, even when withdrawal symptoms are properly recognized, their clinical management is hindered by the complexity of the involved variables and the lack of appropriate research. Reassuring patients that the symptoms are likely to be short-lived and pose no threat [26] is certainly a first helpful step. However, when symptoms persist and are distressing, the reinstitution of the same $\mathrm{AD}$ that triggered the problem or its substitution with a long-acting SSRI such as fluoxetine [26] may only postpone and aggravate the issue [86]. There are anecdotal reports on the use of clonazepam and other benzodiazepines or mood stabilizers, which, however, need to be properly tested $[15,50,59,62,72]$. Another neglected area of research is concerned with the role of withdrawal symptoms in assessing switching strategies that are increasingly utilized in the treatment resistance of mood and anxiety disorders $[86,91]$. Drug effects are attributed without adequate consideration of the confounding role of withdrawal symptoms. Similarly, trial designs, such as in a recent paper on $\mathrm{AD}$ in dementia [92], that assess the effects of discontinuation of AD for inferring efficacy (i.e., a significant increase in depressive symptoms in the patients whose $\mathrm{AD}$ are discontinued compared to those who continue with treatment) are flawed by a lack of consideration of withdrawal events. 
The term 'discontinuation syndrome' has progressively replaced 'withdrawal syndrome' in the SSRI literature. This shift was heavily supported by the pharmaceutical industry $[27,88]$ and was aimed at emphasizing that SSRI do not cause addiction or dependence, and symptoms are substantially different from the phenomena that take place with benzodiazepines [24]. However, Nielsen et al. [27] have demonstrated that there are impressive similarities between SSRI and benzodiazepines in this respect. Defining 'withdrawal syndromes' as those pertaining to benzodiazepines [27], antipsychotic drugs [93], and tricyclic antidepressants [80] and 'discontinuation syndromes' as those of SSRI does not appear to reflect the evidence in the literature. Indeed, the use of this latter term has minimized the potential vulnerabilities induced by SSRI and has provided the ground for misleading in- dications (e.g., $\mathrm{AD}$ are to be preferred to benzodiazepines in anxiety disorders due to a lack of dependence) [94, 95].

Clinicians are familiar with the withdrawal phenomena that may occur from alcohol, benzodiazepines, barbiturates, opioids, and stimulants [96]. The results of this review indicate that they need to add SSRI to the list of drugs potentially inducing withdrawal phenomena. The term 'discontinuation syndrome' minimizes the vulnerabilities induced by SSRI and should be replaced by 'withdrawal syndrome'.

\section{Disclosure Statement}

No author has financial arrangements that might represent potential conflicts of interest for the findings presented.

\section{References}

$>1$ Stoukides J, Stoukides C: Extrapyramidal 14 Leiter F, Nierenberg A, Sanders K, Stern T: symptoms upon discontinuation of fluoxetine. Am J Psychiatry 1991;148:1263.

$>2$ Szabadi E: Fluvoxamine withdrawal syn- $>_{15}$ drome. Br J Psychiatry 1992;160:283-284.

$>3$ Black D, Wesner R, Gabel J: The abrupt discontinuation of fluvoxamine in patients with panic disorder. J Clin Psychiatry 1993;54: 146-149.

4 Barr L, Goodman W, Price L: Physical symptoms associated with paroxetine discontinuation. Am J Psychiatry 1994;151:289.

$\checkmark 5$ Einbinder E: Fluoxetine withdrawal? Am J Psychiatry 1995;152:1253.

6 Debattista C, Schatzberg A: Physical symptoms associated with paroxetine withdrawal. Am J Psychiatry 1995;152:1235-1236.

7 Dominguez R, Goodnick P: Adverse events after the abrupt discontinuation of parox etine. Pharmacotherapy 1995;15:778-780.

$>8$ Bloch M, Stager S, Braun A, Rubinow D: Severe psychiatric symptoms associated with paroxetine withdrawal. Lancet 1995;346:57.

$>9$ Frost L, Lal S: Shock-like sensations after discontinuation of selective serotonin reuptake inhibitors. Am J Psychiatry 1995;152:810.

$>10$ Kasantikul D: Reversible delirium after discontinuation of fluoxetine. J Med Assoc Thai 1995;78:53-54.

$>11$ Phillips S: A possible paroxetine withdrawal syndrome. Am J Psychiatry 1995;152:645646.

-12 Pyke R: Paroxetine withdrawal syndrome. Am J Psychiatry 1995;152:149-150.

-13 Fava G, Grandi S: Withdrawal syndromes after paroxetine and sertraline discontinuation. J Clin Psychopharmacol 1995;15:374-375. Discontinuation reaction following sertraline. Biol Psychiatry 1995;38:694-695.

15 Pacheco L, Malo P, Aragues E: More cases of paroxetine withdrawal syndrome. Br J Psychiatry 1996;169:384.

16 Rosenstock H: Sertraline withdrawal in two brothers: a case report. Int Clin Psychopharmacol 1996;11:58-59.

17 Zajecka J, Fawcett J, Amsterdam J: Safety of abrupt discontinuation of fluoxetine: a randomized, placebo-controlled study. J Clin Psychopharmacol 1998;18:193-197.

18 Lejoyeux M, Adès J: Antidepressant discontinuation: a review of the literature. J Clin Psychiatry 1997;58:11-16.

19 Medawar C: The antidepressant web. Int J Risk Safety Med 1997;10:75-126.

20 Therrier F, Markowitz J: Selective serotonin reuptake inhibitors and withdrawal symptoms: a review of the literature. Hum Psychopharmacol 1997;12:309-323.

21 Olver JS, Burrows GD, Norman TR: Discontinuation syndromes with selective serotonin reuptake inhibitors. CNS Drugs 1999;12:171177.

22 Rosenbaum J, Fava M, Hoog S, Ascroft R, Krebs W: Selective serotonin reuptake inhibitors discontinuation syndrome: a randomized clinical trial. Biol Psychiatry 1998;44:7787.

23 Black K, Shea C, Dursun S, Kutcher S: Selective serotonin reuptake inhibitor discontinuation syndrome: proposed diagnostic criteria. J Psychiatr Neurosci 2000;25:255-261.

24 Shelton R: The nature of the discontinuation syndrome associated with antidepressant drugs. J Clin Psychiatry 2006;67:3-7.
25 Warner C, Bobo W, Warner C, Reid S, Rachal $\mathrm{J}$ : Antidepressant discontinuation syndrome. Am Fam Physician 2006;74:449-456.

26 Schatzberg AF, Blier P, Delgado P, Fava M, Haddad PM, Shelton RC: Antidepressant discontinuation syndrome. J Clin Psychiatry 2006;67:27-30.

27 Nielsen M, Hansen E, Gotzsche P: What is the difference between dependence and withdrawal reactions? A comparison of benzodiazepines and selective serotonin re-uptake inhibitors. Addiction 2012;107:900-908.

28 Renoir T: Selective serotonin reuptake inhibitor antidepressant treatment discontinuation syndrome: a review of the clinical evidence and the possible mechanisms involved. Front Pharmacol 2013;4:1-10.

29 Moher D, Liberati A, Tetzlaff J, Altman DG: Preferred reporting items for systematic reviews and meta-analyses: the PRISMA statement. J Clin Epidemiol 2009;62:1006-1012.

>30 Belaise C, Gatti A, Chouinard VA, Chouinard G: Patient online report of selective serotonin reuptake inhibitor induced persistent postwithdrawal anxiety and mood disorders. Psychother Psychosom 2012;81:386-388.

31 Chouinard G: Issues in the clinical use of benzodiazepines: potency, withdrawal and rebound. J Clin Psychiatry 2004;65 (suppl 5): 7-12.

32 Oehrberg S, Christiansen PE, Behnke K, Borup AL, Severin B, Soegaard J, Calberg H, Judge R, Ohrstrom JK, Manniche PM: Paroxetine in the treatment of panic disorder: a randomized, double-blind, placebo-controlled study. Br J Psychiatry 1995;167:374-379. 
33 Markowitz J, DaVane C, Liston H, Montgomery S: An assessment of selective serotonin reuptake inhibitor discontinuation symptoms with citalopram. Int Clin Psychopharmacol 2000;15:329-333.

- 34 Robert P, Montgomery SA: Citalopram in doses of 20-60 mg is effective in depression relapse prevention: a placebo-controlled 6-month study. Int Clin Psychopharmacol 1995;10:29-35.

- 35 Lingjaerde O, Ahlfors UG, Bech P, Dencker SJ, Elgen K: The UKU side effect rating scale. A new comprehensive rating scale for psychotropic drugs and a cross-sectional study of side effects in neuroleptic-treated patients. Acta Psychiatr Scand 1987;334:1-100.

- 36 Montgomery S, Nil R, Durr-Pal N, Loft H, Boulenger J: A 24-week randomized, doubleblind, placebo-controlled study of escitalopram for the prevention of generalized social anxiety disorder. J Clin Psychiatry 2005;66: 1270-1278.

37 Michelson D, Fava M, Amsterdam J, Apter J, Londborg P, Tamura R, Tepner R: Interruption of selective serotonin reuptake inhibitor treatment. Double-blind placebo-controlled trial. Br J Psychiatry 2000;176:363-368.

38 Judge R, Parry M, Quail D, Jacobson J: Discontinuation symptoms: comparison of brief interruption in fluoxetine and paroxetine treatment. Int Clin Psychopharmacol 2002; $17: 217-225$

-39 Montgomery S, Kennedy S, Lejoyeux M, Hindmarch I: Absence of discontinuation symptoms with agomelatine and occurrence of discontinuation symptoms with paroxetine: a randomized, double-blind, placebocontrolled discontinuation study. Int Clin Psychopharmacol 2004;19:271-280.

40 Hindmarch I, Kimber S, Cocle S: Abrupt and brief discontinuation of antidepressant treatment: effects on cognitive function and psychomotor performance. Int Clin Psychopharmacol 2000;15:305-318.

41 Montgomery S, Huusom A, Bothmer J: A randomised study comparing escitalopram with venlafaxine XR patients in primary care with major depressive disorder. Neuropsychobiology 2004;50:57-64.

-42 Sir A, D'Souza RF, Uguz S, George T, Vahip S, Hopwood M, Martin AJ, Lam W, Burt T: Randomized trial of sertraline versus venlafaxine XR in major depression: efficacy and discontinuation symptoms. J Clin Psychiatry 2005;66:1312-1320.

43 Lader M, Stender K, Burger V, Nil R: Efficacy and tolerability of escitalopram in 12- and 24week treatment of social anxiety disorder: a randomized, double-blind, placebo-controlled, fixed-dose study. Depress Anxiety 2004; 19:241-248.

44 Baldwin D, Huusom A, Maehlum E: Escitalopram and paroxetine in the treatment of generalised anxiety disorder: randomised placebo-controlled, double-blind study. Br J Psychiatry 2006;189:264-272.
45 Baldwin D, Cooper J, Huusom A, Hindmarch I: A double-blind, randomized, parallelgroup, flexible-dose study to evaluate the tolerability, efficacy and effects of treatment discontinuation with escitalopram and paroxetine in patients with major depressive disorder. Int Clin Psychopharmacol 2006;21: 159-169.

46 Tint A, Haddad P, Anderson I: The effect of rate of antidepressant tapering on the incidence of discontinuation symptoms: a randomized study. J Psychopharmacol 2008;22: 330-332.

47 Haddad P, Lejoyeux M, Young A: Antidepressant discontinuation reactions are preventable and simple to treat. BMJ 1998;316: 1105-1106.

48 Mallya G, White K, Gunderson C: Is there a serotoninergic withdrawal syndrome? Biol Psychiatry 1993;33:851-852.

49 Bogetto F, Bellino S, Bonatto R, Patria L: Discontinuation syndrome in dysthymic patients treated with selective serotonin reuptake inhibitors: a clinical investigation. CNS Drugs 2002; 16:273-283.

50 Fava G, Bernardi M, Tomba E, Rafanelli C: Effects of gradual discontinuation of selective serotonin reuptake inhibitors in panic disorder with agoraphobia. Int J Neuropsychopharmacol 2007;10:835-838.

51 American Psychiatric Association: Diagnostic and Statistical Manual of Mental Disorders, ed 4. Washington, American Psychiatric Association, 1994.

52 Coupland NJ, Bell C, Potokar JP: Serotonin reuptake inhibitor withdrawal. J Clin Psychopharmacol 1996;16:356-362.

53 Price J, Waller P, Wood S, Mackay A: A comparison of the post-marketing safety of four selective serotonin reuptake inhibitors including the investigation of symptoms occurring on withdrawal. Br J Clin Pharmacol 1996; 42:757-763.

54 Himei A, Okamura T: Discontinuation syndrome associated with paroxetine in depressed patients: a retrospective analysis of factor involved in the occurrence of the syndrome. CNS Drugs 2006;20:665-672.

-55 Van Geffen E, Hugtenburg J, Heerdink E, Van Hulten R, Egberts A: Discontinuation symptoms in users of selective serotonin reuptake inhibitors in clinical practice: tapering versus abrupt discontinuation. Eur J Clin Psychopharmacol 2005;61:303-307.

56 Cooper G: The safety of fluoxetine: an update. Br J Psychiatry 1988;153:77-86.

57 Poryazova R, Siccoli M, Werth E, Bassetti C: Unusually prolonged rebound cataplexy after withdrawal of fluoxetine. Neurology 2005;65: 967-968.

58 Blum D, Maldonado J, Meyer E, Lanberg M: Delirium following abrupt discontinuation of fluoxetine. Clin Neurol Neurosurg 2008;110: 69-70.
59 Bhanji N, Chouinard G, Kolivakis T, Margolese H: Persistent tardive rebound panic disorder, rebound anxiety and insomnia following paroxetine withdrawal: a review of rebound withdrawal phenomena. Can J Clin Pharmacol 2006;13:69-74.

60 Keuthen N, Cyr P, Ricciardi J: Medication withdrawal symptoms in obsessive-compulsive disorder patients treated with paroxetine. Int Clin Psychopharmacol 1994;14:206-207.

61 Milliken C, Cooper S: Withdrawal symptoms from paroxetine. Hum Psychopharmacol 1998;13:217-219.

62 Belaise C, Gatti A, Chouinard VA, Chouinard G: Persistent post-withdrawal disorders induced by paroxetine, a selective serotonin reuptake inhibitor, and treated with specific cognitive behavioral therapy. Psychother Psychosom 2014;83:247-248.

63 Yasui-Furukori N, Kaneko S: Hallucination induced by paroxetine discontinuation in patients with major depressive disorders. Psychiatry Clin Neurosci 2011;65:384-385.

64 Polimeni G, Grugno R, Cordici F, Vietta A, Caputi A, Bramanti P: Nocturnal enuresis after paroxetine abrupt discontinuation: a case report. J Clin Psychopharmacol 2008;28:589591.

65 Mazzatenta C, Peonia G, Martini P: Pruritus induced by interruption of paroxetine therapy. Br J Dermatol 2004;150:787.

66 Landry P, Roy L: Withdrawal hypomania associated with paroxetine. J Clin Psychopharmacol 1997;17:60-61.

67 Shoenberger D: Discontinuing paroxetine: a personal account. Psychother Psychosom 2002;71:237-238.

68 Louie A, Lannon R, Ajari L: Withdrawal reaction after sertraline discontinuation. Am J Psychiatry 1994;151:450.

69 Levenson J: Putative neuroleptic malignant syndrome associated with sertraline withdrawal. J Clin Psychopharmacol 2009;29:300-301.

70 Benazzi F: Psychotic mania in bipolar II depression related to sertraline discontinuation. Can J Psychiatry 2002;47:584-585.

71 Amsden G, Georgian F: Orthostatic hypotension induced by sertraline withdrawal. Pharmacotherapy 1996;16:684-686.

72 Benazzi F: SSRI discontinuation syndrome related to fluvoxamine. Rev Psychiatr Neurosci 1998;23:94.

73 Hirose S: Restlessness related to SSRI withdrawal. Psychiatry Clin Neurosci 2001;55:7780.

74 Benazzi F: Citalopram withdrawal symptoms. Eur Psychiatry 1998;13:219.

75 Fernando A, Schwader P: A case of citalopram withdrawal. J Clin Psychopharmacol 2000;20: 581-582.

76 Adson D, Kotlyar M: Premature ejaculation associated with citalopram withdrawal. Ann Pharmacother 2003;37:1804-1806.

-77 Feth N, Cattapan-Ludewig K, JaquenoudSirot E: Electric sensations: neglected symptom of escitalopram discontinuation. Am J Psychiatry 2006;163:160. 
78 Prakash O, Dhar V: Emergence of electric shock-like sensations on escitalopram discontinuation. J Clin Psychopharmacol 2008; 28:359-360.

79 Tomba E, Offidani E, Fava GA: Selective serotonin reuptake inhibitors withdrawal syndromes: new insights into pathophysiology and treatment; in Rees JP, Woodhouse $\mathrm{OB}$ (eds): Substance Withdrawal Syndrome. New York, Nova Science, 2009, pp 37-60.

$>80$ Bitter I, Filipovits D, Czobor P: Adverse reactions to duloxetine in depression. Expert Opin Drug Saf 2011;10:839-850.

-81 Dilsaver SC, Greden JF: Antidepressant withdrawal phenomena. Biol Psychiatry 1984;19: 237-256.

82 Blier P, Tremblay P: Physiologic mechanisms underlying the antidepressant discontinuation syndrome. J Clin Psychiatry 2006;67:813.

83 Harvey BH, McEwen BS, Stein DJ: Neurobiology of antidepressant withdrawal: implications for longitudinal outcome of depression. Biol Psychiatry 2003;54:1105-1117.
84 Murata Y, Kobayashi D, Imuta N, Haraguchi K, Ieiri I, Nishimura R, Koyama S, Mine K: Effects of serotonin 1A, 2A, 2C, 3A and $3 \mathrm{~B}$ and serotonin transporter gene polymorphisms on the occurrence of paroxetine discontinuation syndrome. J Clin Psychopharmacol 2010;30:11-17.

85 Fava GA, Offidani E: The mechanisms of tolerance in antidepressant action. Prog Neuropsychopharmacol Biol Psychiatry 2011;35: 1593-1602.

86 Fava GA: Rational use of antidepressant drugs. Psychother Psychosom 2014;83:197204.

87 Offidani E, Guidi J, Tomba E, Fava GA: Efficacy and tolerability of benzodiazepines versus antidepressants in anxiety disorders: a systematic review and meta-analysis. Psychother Psychosom 2013;82:355-362.

88 Fava GA, Tomba E: The use of antidepressant drugs: some reasons for concern. Int J Risk Safety Med 1998;11:271-274.

89 Fava GA, Tomba E, Tossani E: Innovative trends in the design of therapeutic trials in psychopharmacology and psychotherapy. Prog Neuropsychopharmacol Biol Psychiatry 2013;40:306-311.
90 Offidani E, Fava GA, Sonino N: Iatrogenic comorbidity in childhood and adolescence: new insights from the use of antidepressant drugs. CNS Drugs 2014;28:769-774.

91 Carvalho AF, Berk M, Hyphantis TN, McIntyre RS: The integrative management of treatment resistant depression. Psychother Psychosom 2014;83:70-88.

92 Bergh S, Selbaek G, Engedal K: Discontinuation of antidepressants in people with dementia and neuropsychiatric symptoms (DESEP Study). BMJ 2012;344:1566.

93 Cerovecki A, Musil R, Klimke A, Seemuller F, Haen E, Schennach R, Kuhn KU, Volz HP, Riedel M: Withdrawal symptoms and rebound syndromes associated with switching and discontinuing atypical antipsychotics. CNS Drugs 2013;27:545-572.

94 Rickels K: Should benozodiazepines be replaced by antidepressants in the treatment of anxiety disorders? Fact or fiction? Psychother Psychosom 2013;82:351-352.

95 Balon R: Benzodiazepines revisited. Psychother Psychosom 2013;82:353-354.

96 Kosten TR, O’Connor PG: Management of drug and alcohol withdrawal. N Engl J Med 2003;348:1786-1795. 